Power filtering of $n$th order in the fractional Fourier domain

This content has been downloaded from IOPscience. Please scroll down to see the full text. 2002 J. Phys. A: Math. Gen. 357779

(http://iopscience.iop.org/0305-4470/35/36/306)

View the table of contents for this issue, or go to the journal homepage for more

Download details:

IP Address: 147.96.14.16

This content was downloaded on 30/01/2014 at 18:32

Please note that terms and conditions apply. 


\title{
Power filtering of $\boldsymbol{n}$ th order in the fractional Fourier domain
}

\author{
Tatiana Alieva $^{1}$, Maria Luisa Calvo ${ }^{1}$ and Martin $\mathrm{J} \mathrm{Bastiaans}^{2}$ \\ ${ }^{1}$ Departamento de Óptica, Facultad de Físicas, Universidad Complutense de Madrid, \\ 28040 Madrid, Spain \\ ${ }^{2}$ Faculteit Elektrotechniek, Technische Universiteit Eindhoven, Postbus 513, \\ 5600 MB Eindhoven, The Netherlands
}

Received 12 April 2002, in final form 19 June 2002

Published 28 August 2002

Online at stacks.iop.org/JPhysA/35/7779

\begin{abstract}
The main properties of the power filtering operation in the fractional Fourier domain and its relationship to the differentiation operation are considered. The application of linear power filtering for solving the phase retrieval problem from intensity distributions only is proposed. The optical configuration for the experimental realization of the method is discussed.
\end{abstract}

PACS numbers: $\quad 02.30 . \mathrm{Nw}, 42.30 . \mathrm{Kq}$

The usefulness of the Fourier transform (FT) is often related to the simplification of the differentiation operation, which permits one to solve a number of differential equations. Thus, power filtering in the Fourier domain is related to the signal derivative as

$$
R^{-\pi / 2}\left[F_{\pi / 2}(u) u^{n}\right](x)=(-\mathrm{i})^{n} \frac{\mathrm{d}^{n} f(x)}{\mathrm{d} x^{n}}
$$

where $F_{\pi / 2}(u)$ is the FT of $f(x)$ and $R^{-\pi / 2}[\cdot]$ denotes the inverse Fourier operation, cf equation (2). Also note that the first and the second derivatives (or power filtering for $n=1,2$ ) are widely used for edge characterization.

The introduction of the fractional FT to quantum mechanics, paraxial optics and signal/image processing [1-4] has permitted the simplification of the description of the corresponding systems and the development of new methods for signal analysis [5]. Thus, the fractional correlation and convolution operations related to filtering in the fractional Fourier domain were extensively studied previously [6-8]. Nevertheless, the question of power filtering in the fractional Fourier domain and its relationship to the differentiation operation does not seem to have been considered in the literature and is the subject of this paper.

The fractional FT is a generalization of the ordinary FT. Its kernel depends on a parameter that can be interpreted as a rotation angle in phase space. The fractional FT of a function $f(x)$ for an angle $\alpha$ is defined as [4]

$$
R^{\alpha}[f(x)](u)=F_{\alpha}(u)=\int_{-\infty}^{\infty} f(x) K(\alpha, x, u) \mathrm{d} x
$$


where the kernel is given by

$$
K(\alpha, x, u)=\frac{\exp (\mathrm{i} \alpha / 2)}{\sqrt{\mathrm{i} 2 \pi \sin \alpha}} \exp \left[\mathrm{i} \frac{\left(x^{2}+u^{2}\right) \cos \alpha-2 x u}{2 \sin \alpha}\right] .
$$

The kernel of the fractional FT is periodic in $\alpha$. For $\alpha=0$ the fractional FT corresponds to the identity operation, $F_{0}(u)=f(u)$, and for $\alpha=\pi / 2$ and $3 \pi / 2$ it reduces to the FT and the inverse FT, respectively; moreover $F_{\pi}(u)=f(-u)$. The fractional FT is additive with respect to $\alpha, R^{\alpha+\beta}=R^{\alpha} R^{\beta}$, and-as can easily be seen from equation (3)-the kernel $K(-\alpha, x, u)$ of the inverse fractional FT, $R^{-\alpha}$ is equal to $K^{*}(\alpha, x, u)$.

The kernel of the fractional FT is, except for a phase shift $\alpha / 2$, a propagator of the non-stationary Schrödinger equation for a harmonic oscillator (here dimensionless variables are used):

$$
\left[\frac{\partial}{\partial \alpha}-\frac{i}{2} \frac{\partial^{2}}{\partial x^{2}}+\frac{i}{2} x^{2}\right] \Psi(x, \alpha)=0 .
$$

The same equation describes in the paraxial approximation of scalar diffraction theory the complex field amplitude $\Psi(r, z)$ during its propagation through a medium with a quadratic index of refraction $n^{2}=n_{0}^{2}\left(1-g^{2} r^{2}\right)[3,5]$. In this case we have $\alpha=g z$ and $x=\sqrt{k g} r$, where $r$ and $z$ are the transversal and longitudinal coordinates, respectively, and $k$ is the wavenumber. The fractional FT of the complex field amplitude as well as the ordinary FT can also be performed by using a thin lens with focal distance $\mathbf{f}$, with the input and output planes located at distances $d=2 \mathbf{f} \sin ^{2} \alpha / 2$ before and after the lens, respectively [9].

Based on the additivity property of the fractional FT, equation (1) can easily be generalized as

$$
R^{-\pi / 2}\left[F_{\alpha+\pi / 2}(u) u^{n}\right](x)=(-\mathrm{i})^{n} \frac{\mathrm{d}^{n} F_{\alpha}(x)}{\mathrm{d} x^{n}} .
$$

Then the ordinary $n$-power filtering operation performed in any $\beta=\alpha+\pi / 2$ fractional Fourier domain is related to the $n$th derivative of the $\alpha$-fractional FT. Moreover, from the additivity property it also follows that $R^{\beta}\left[F_{\alpha}(u) g(u)\right](x)=R^{\beta+\alpha}\left[R^{-\alpha}\left[F_{\alpha}(u) g(u)\right](y)\right](x)$ with, in our case, $g(u)=u^{n}$.

In this paper we restrict ourselves to considering the operation $R^{-\alpha}\left[F_{\alpha}(u) u^{n}\right](x)$, because it corresponds to a logical generalization of $n$ th-order power filtering (see equation (1)) in the fractional FT domain. Note that the operation $R^{\alpha}\left[f(u) u^{n}\right](x)$ for different integers $n$ was considered in $[1,2,4]$. Since

$$
\begin{aligned}
& \int K(-\alpha, x, u) K(\alpha, \xi, u) u^{n} \mathrm{~d} u=(-\mathrm{i} \sin \alpha)^{n} \exp \left[-\mathrm{i}\left(x^{2}-\xi^{2}\right) \cot \alpha / 2\right] \delta^{(n)}(x-\xi) \\
& \text { We have } \\
& \begin{aligned}
R^{-\alpha}\left[F_{\alpha}(u) u^{n}\right](x)=\int_{-\infty}^{\infty} K(-\alpha, x, u) F_{\alpha}(u) u^{n} \mathrm{~d} u \\
\qquad \int_{-\infty}^{\infty} f(\xi) \mathrm{d} \xi \int_{-\infty}^{\infty} K(-\alpha, x, u) K(\alpha, \xi, u) u^{n} \mathrm{~d} u \\
=(-\mathrm{i} \sin \alpha)^{n} \exp \left(-\mathrm{i} x^{2} \cot \alpha / 2\right) \frac{\mathrm{d}^{n}}{\mathrm{~d} x^{n}} f(x) \exp \left(\mathrm{i} x^{2} \cot \alpha / 2\right) \\
=(-\mathrm{i} \sin \alpha)^{n} \exp \left(-\mathrm{i} x^{2} \cot \alpha / 2\right) \sum_{k=0}^{n}\left(\begin{array}{l}
n \\
k
\end{array}\right) \frac{\mathrm{d}^{n-k} \exp \left(\mathrm{i} x^{2} \cot \alpha / 2\right)}{\mathrm{d} x^{n-k}} \frac{\mathrm{d}^{k} f(x)}{\mathrm{d} x^{k}} \\
=(\mathrm{i} \sin \alpha)^{n} \sum_{k=0}^{n}\left(\begin{array}{l}
n \\
k
\end{array}\right)(-1)^{k}(\sqrt{\cot \alpha / 2 \mathrm{i}})^{n-k} H_{n-k}(x \sqrt{\cot \alpha / 2 \mathrm{i}}) \frac{\mathrm{d}^{k} f(x)}{\mathrm{d} x^{k}}
\end{aligned}
\end{aligned}
$$


where $H_{n}(z)=(-1)^{n} \exp \left(z^{2}\right) \mathrm{d}^{n} \exp \left(-z^{2}\right) / \mathrm{d} z^{n}$ are the Hermite polynomials [10]. This equation is a generalization of equation (1) in the fractional Fourier domain. Alternately, based on the multiplication rule derived in [1, 2], $R^{\alpha}\left[f(u) u^{n}\right](x)=(x \cos \alpha+$ i $\sin \alpha \mathfrak{D})^{n} R^{\alpha}[f(u)](x)$, the $n$ th-order power filtering operation can be written in the form

$$
R^{-\alpha}\left[F_{\alpha}(u) u^{n}\right](x)=(x \cos \alpha-\mathrm{i} \sin \alpha \mathfrak{D})^{n} f(x)
$$

where $\mathfrak{D}$ denotes the differential operator $d / d x$. It is easy to see from equations (5) and (6) that

$$
R^{-\alpha}\left[F_{\alpha}(u) u^{n}\right](x)=(-1)^{n} R^{-\alpha-\pi}\left[F_{\alpha+\pi}(u) u^{n}\right](x) .
$$

Moreover, for real $f(x)$ we find that $\left\{R^{-\alpha}\left[F_{\alpha}(u) u^{n}\right](x)\right\}^{*}=R^{\alpha}\left[F_{-\alpha}(u) u^{n}\right](x)$, and therefore $\left|R^{-\alpha}\left[F_{\alpha}(u) u^{n}\right](x)\right|^{2}=\left|R^{\alpha}\left[F_{-\alpha}(u) u^{n}\right](x)\right|^{2}$.

We start with the case of $n=1$, which is related to the first derivative of the signal $f(x)$. Filtering in the fractional Fourier domain with mask $u$ yields the following result

$$
\begin{aligned}
& R^{\mp \alpha}\left[F_{ \pm \alpha}(u) u\right](x)=\cos \alpha x f(x) \mp \mathrm{i} \sin \alpha \frac{\mathrm{d} f(x)}{\mathrm{d} x} \\
& =\cos \alpha R^{0}\left[F_{0}(u) u\right](x) \pm \sin \alpha R^{-\pi / 2}\left[F_{\pi / 2}(u) u\right](x)
\end{aligned}
$$

which can be considered as a weighted sum of corresponding filtering results in the position and Fourier domains.

Taking equation (8) for two different angles $\alpha$ and $\beta$, we can find the first derivative $\mathrm{d} f / \mathrm{d} x=\mathrm{i} R^{-\pi / 2}\left[F_{\pi / 2}(u) u\right](x)$ and the product $x f(x)=R^{0}\left[F_{0}(u) u\right](x)$ as:

$-\mathrm{i} \frac{\mathrm{d} f(x)}{\mathrm{d} x}=\frac{1}{\sin (\alpha-\beta)}\left\{\cos \beta R^{-\alpha}\left[F_{\alpha}(u) u\right](x)-\cos \alpha R^{-\beta}\left[F_{\beta}(u) u\right](x)\right\}$

$x f(x)=\frac{-1}{\sin (\alpha-\beta)}\left\{\sin \beta R^{-\alpha}\left[F_{\alpha}(u) u\right](x)-\sin \alpha R^{-\beta}\left[F_{\beta}(u) u\right](x)\right\}$.

In the particular case of $\beta=\alpha+\pi / 2$, equations (9) can be written in the form of a matrix-vector product as

$$
\left(\begin{array}{c}
R^{-\pi / 2}\left[F_{\pi / 2}(u) u\right](x) \\
R^{0}\left[F_{0}(u) u\right](x)
\end{array}\right)=\left(\begin{array}{cc}
\cos \alpha & \sin \alpha \\
-\sin \alpha & \cos \alpha
\end{array}\right)\left(\begin{array}{c}
R^{-\alpha-\pi / 2}\left[F_{\alpha+\pi / 2}(u) u\right](x) \\
R^{-\alpha}\left[F_{\alpha}(u) u\right](x)
\end{array}\right) .
$$

The two expressions on the right-hand side of equation (10) are invariant with respect to $\alpha$, and the same holds for the sum of the intensities in two Fourier-conjugated domains:

$$
\left|R^{-\alpha-\pi / 2}\left[F_{\alpha+\pi / 2}(u) u\right](x)\right|^{2}+\left|R^{-\alpha}\left[F_{\alpha}(u) u\right](x)\right|^{2}=|x f(x)|^{2}+\left|\frac{\mathrm{d} f(x)}{\mathrm{d} x}\right|^{2} .
$$

For the particular case of $\beta=-\alpha$, or, equivalently, for the case of $\beta=\pi-\alpha$ and using equation (7), equations (9) yield

$$
\begin{aligned}
-\mathrm{i} \frac{\mathrm{d} f(x)}{\mathrm{d} x} & =\frac{1}{2 \sin \alpha}\left\{R^{-\alpha}\left[F_{\alpha}(u) u\right](x)-R^{\alpha}\left[F_{-\alpha}(u) u\right](x)\right\} \\
x f(x) & =\frac{1}{2 \cos \alpha}\left\{R^{-\alpha}\left[F_{\alpha}(u) u\right](x)+R^{\alpha}\left[F_{-\alpha}(u) u\right](x)\right\} .
\end{aligned}
$$

Note that the expressions on the right-hand side of equations (12) are also invariant with respect to $\alpha$.

From equation (8) we conclude that the sum of the squared moduli of the filtered signal (first-order power filtered in the $+\alpha$ and the $-\alpha$ fractional Fourier domain) is related to the squared moduli of the signal derivative and the signal intensity as

$$
\frac{1}{2}\left\{\left|R^{-\alpha}\left[F_{\alpha}(u) u\right](x)\right|^{2}+\left|R^{\alpha}\left[F_{-\alpha}(u) u\right](x)\right|^{2}\right\}=\cos ^{2} \alpha|x f(x)|^{2}+\sin ^{2} \alpha\left|\frac{\mathrm{d} f(x)}{\mathrm{d} x}\right|^{2}
$$




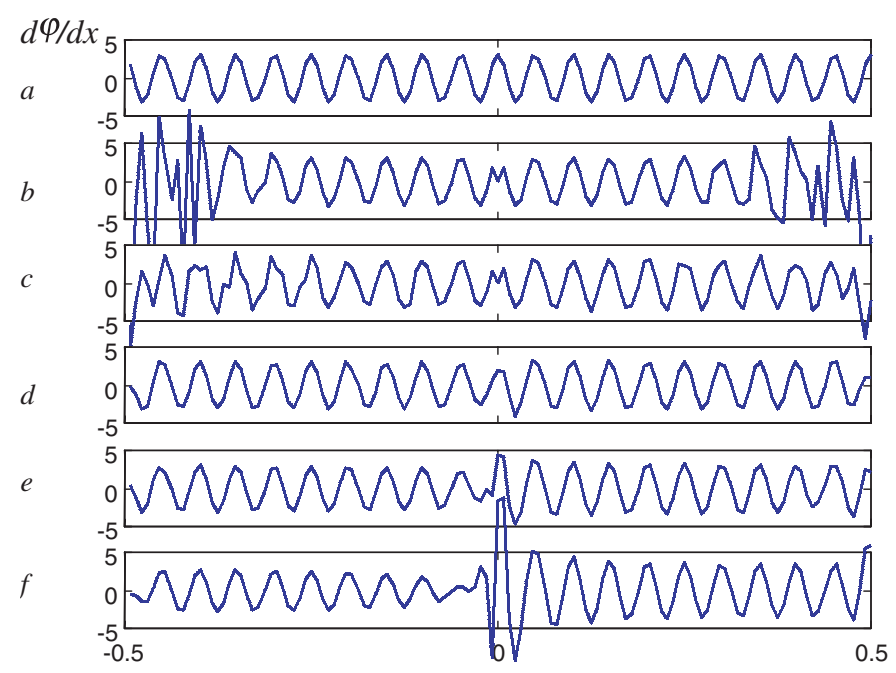

Figure 1. Original and reconstructed phase derivative of the signal $f(x)=\exp \left[-6 x^{2}+\right.$ $\mathrm{i} 0.025 \sin (40 \pi x)]$ for different values of the fractional angle $\alpha:(a) \alpha=0$, original phase derivative; (b) $\alpha=0.1 \pi / 2 ;(c) \alpha=0.25 \pi / 2 ;(d) \alpha=0.5 \pi / 2 ;(e) \alpha=0.75 \pi / 2 ;(f) \alpha=0.9 \pi / 2$.

and that their difference is connected to the amplitude $|f(x)|$ and the phase $\varphi(x)=\arg f(x)$ as

$$
\frac{1}{2 \sin 2 \alpha}\left\{\left|R^{-\alpha}\left[F_{\alpha}(u) u\right](x)\right|^{2}-\left|R^{\alpha}\left[F_{-\alpha}(u) u\right](x)\right|^{2}\right\}=x|f(x)|^{2} \frac{\mathrm{d} \varphi(x)}{\mathrm{d} x} .
$$

We stress again the $\alpha$-invariance of the expression in equation (14). Equation (14) can be applied for solving the phase retrieval problem, at least for $\alpha \neq n \pi / 2$. Indeed, the phase derivative $\mathrm{d} \varphi / \mathrm{d} x$, and therefore the phase $\varphi(x)$ up to a constant term, can be reconstructed from the knowledge of the intensity $|f(x)|^{2}$ and the intensity distributions at the output of two fractional FT filters with mask $u$ :

$$
\frac{\mathrm{d} \varphi(x)}{\mathrm{d} x}=\frac{1}{2 \sin 2 \alpha x|f(x)|^{2}}\left\{\left|R^{-\alpha}\left[F_{\alpha}(u) u\right](x)\right|^{2}-\left|R^{\alpha}\left[F_{-\alpha}(u) u\right](x)\right|^{2}\right\} .
$$

In order to illustrate the efficiency of this method, numerical simulations have been carried out for a signal with Gaussian amplitude and sinusoidal phase $f(x)=$ $\exp \left[-6 x^{2}\right] \exp [\mathrm{i} 0.025 \sin (40 \pi x)]$, where $x \in[-0.5 ; 0.5]$. In figure $1(a)$ we have depicted the original phase derivative, $\pi \cos 40 \pi x$, whereas the reconstructed derivatives - determined using equation (15) — are shown in figure 1(b)- $(f)$ for several values of the fractional angle $\alpha$. The fractional FTs have been calculated following the procedure proposed in [11]. We note that the reconstruction is good for those values of $x$ for which $x|f(x)|^{2}$ is not too small. Note, moreover, that in the central region $x \in[-0.1 ; 0.1]$ the quality of reconstruction is better for small angles, whereas for $|x| \in[0.3 ; 0.5]$ the better results are observed for $\alpha \geqslant \pi / 4$. We have limited the number of sensor points to 128 , in order to make the simulations closer to the experiments. In general, increasing the number of sensor points improves the reconstruction quality.

As it is relatively simple to perform a fractional FT in optics, the proposed method can be used for the phase retrieval of optical fields from intensity distribution data. The hybrid opto-electronic processor realizing the procedure (15) is shown in figure 2. The optical beam is first divided into two parts by a beamsplitter, which then propagate through optical 


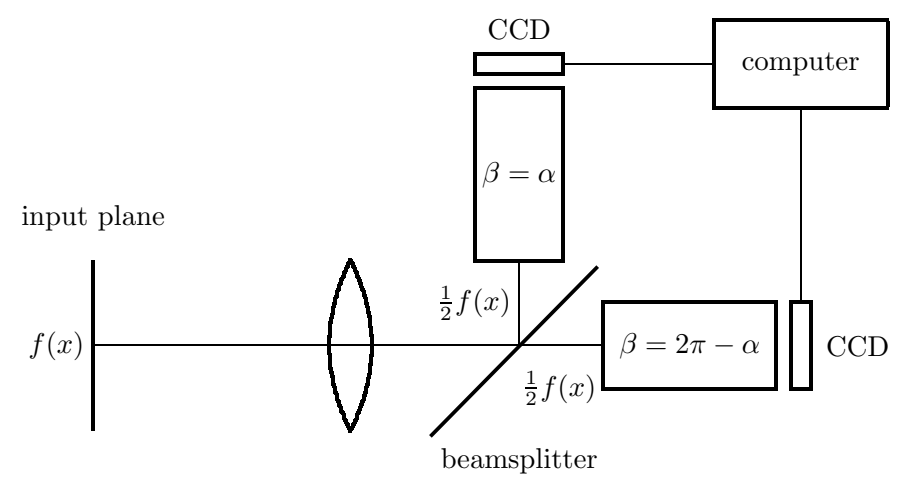

Figure 2. The optical field $f(x)$ in the input plane is imaged through a beamsplitter on to two optical, fractional power-filtering systems (see figure 3) with fractional angles $\beta=\alpha$ and $\beta=2 \pi-\alpha$, the output fields of which are captured by CCD cameras for further electronic processing.

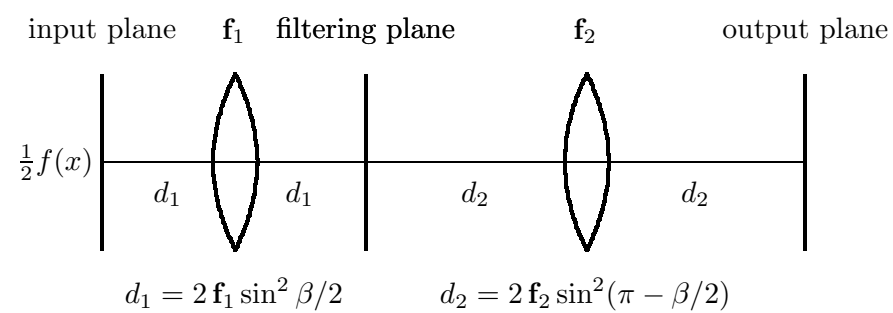

Figure 3. A cascade of a fractional FT system and an inverse fractional FT system, each consisting of one thin lens with focal length $\mathbf{f}_{1,2}$, preceded and followed by two identical distances $d_{1,2}$ of free space. The relations between the distances, the focal lengths and the fractional angle $\beta$ are $d_{1}=2 \mathbf{f}_{1} \sin ^{2} \beta / 2$ and $d_{2}=2 \mathbf{f}_{2} \sin ^{2}(\pi-\beta / 2)$.

systems similar to the one represented in figure 3. The intensity distributions at the output of these systems are registered by CCD cameras and proceed to a computer where additional algebraic operations are made. The optical set-up (see figure 3) proposed for the experimental measurements of the two intensity distributions $\left|R^{-\alpha}\left[F_{\alpha}(u) u\right](x)\right|^{2}$ and $\left|R^{\alpha}\left[F_{-\alpha}(u) u\right](x)\right|^{2}$ consists of two thin lenses with focal distances $\mathbf{f}_{1}$ and $\mathbf{f}_{2}$, and a filter with transfer function $g(u)=u$; negative values of $g(u)$ (for $u<0$ ) are realized by placing a $\pi$ phase step over the negative half of the $u$ plane. The configuration parameters (distances $d_{1}$ and $d_{2}$, and focal distances $\mathbf{f}_{1}$ and $\mathbf{f}_{2}$ of the two lenses) for each of the two optical systems in figure 2 are chosen in such a way that $\beta=\alpha$ for one beam, and $\beta=2 \pi-\alpha$ for the other. The optical set-up in figure 3 can be considered as a cascade of two subsystems, one from the input plane to the filtering plane, and one from the filtering plane to the output plane, with the amplitude plate in the filtering plane. The first subsystem performs a fractional FT for the angle $\beta$, if the relation $d_{1}=2 \mathbf{f}_{1} \sin ^{2} \beta / 2$ is satisfied [9]; similarly, the second subsystem performs an inverse fractional FT, if the relation $d_{2}=2 \mathbf{f}_{2} \sin ^{2}(\pi-\beta / 2)$ holds. Thus, the optical field $f(x) / 2$ is first fractional Fourier transformed to yield $F_{\beta}(u) / 2=R^{\beta}[f(x) / 2](u)$ just before the filtering mask. After passing through the filter, the optical field reads $F_{\beta}(u) u / 2$. Finally, the inverse fractional FT system yields in its output plane the optical field $R^{-\beta}\left[F_{\beta}(u) u / 2\right](x)$, the intensity distribution of which is registered by a CCD camera. 
Linear filtering in the fractional Fourier domain can thus be used for the phase retrieval of optical fields. Note that the proposed method is non-interferometric and non-iterative.

Let us now consider equation (5) for the case of $n=2$, which is related to the second derivative of the signal $f(x)$. Filtering in the fractional Fourier domain with mask $u^{2}$ yields (cf equation (8))

$R^{\mp \alpha}\left[F_{ \pm \alpha}(u) u^{2}\right](x)=\cos ^{2} \alpha x^{2} f(x)-\sin ^{2} \alpha \frac{\mathrm{d}^{2} f(x)}{\mathrm{d} x^{2}} \mp \mathrm{i} \cos \alpha \sin \alpha\left[f(x)+2 x \frac{\mathrm{d} f(x)}{\mathrm{d} x}\right]$

and hence

$\frac{1}{2}\left\{R^{-\alpha}\left[F_{\alpha}(u) u^{2}\right](x)+R^{\alpha}\left[F_{-\alpha}(u) u^{2}\right](x)\right\}=\cos ^{2} \alpha x^{2} f(x)-\sin ^{2} \alpha \frac{\mathrm{d}^{2} f(x)}{\mathrm{d} x^{2}}$.

If we take the latter relationship for two different angles $\alpha$ and $\beta$, we can find the second derivative $\mathrm{d}^{2} f / \mathrm{d} x^{2}$ as (cf equation (9))

$$
\begin{gathered}
\frac{\mathrm{d}^{2} f(x)}{\mathrm{d} x^{2}}=\frac{1}{2\left(\cos ^{2} \beta-\cos ^{2} \alpha\right)}\left\{\cos ^{2} \alpha\left(R^{-\beta}\left[F_{\beta}(u) u^{2}\right](x)+R^{\beta}\left[F_{-\beta}(u) u^{2}\right](x)\right)\right. \\
\left.-\cos ^{2} \beta\left(R^{-\alpha}\left[F_{\alpha}(u) u^{2}\right](x)+R^{\alpha}\left[F_{-\alpha}(u) u^{2}\right](x)\right)\right\} .
\end{gathered}
$$

If we combine equation (16) for the angle $\alpha$ with a similar expression for the angle $\alpha+\pi / 2$, we obtain the relationship

$R^{-\alpha-\pi / 2}\left[F_{\alpha+\pi / 2}(u) u^{2}\right](x)+R^{-\alpha}\left[F_{\alpha}(u) u^{2}\right](x)=x^{2} f(x)-\frac{\mathrm{d}^{2} f(x)}{\mathrm{d} x^{2}}$,

from which we conclude that the sum $R^{-\alpha-\pi / 2}\left[F_{\alpha+\pi / 2}(u) u^{2}\right](x)+R^{-\alpha}\left[F_{\alpha}(u) u^{2}\right](x)$ is invariant with respect to $\alpha$.

We have derived a general expression for the power filtering of $n$th order in the fractional Fourier domain, which stresses its relation to the differentiation operation. The main properties and invariants of linear and quadratic fractional filtering have been found. In particular, it has been shown that the signal derivative and the corresponding power filtering in the Fourier domain, can be represented as a linear combination of the related fractional power filtering operations. The application of linear filtering in the fractional Fourier domain for phase retrieval from intensity distributions only has been proposed. Its efficiency has been demonstrated by numerical simulations. A simple optical configuration for the experimental realization of the method has been discussed.

\section{Acknowledgments}

Financial assistance from the multidisciplinary project PR486/97-7477/97 from Complutense University of Madrid and grant TIC2000-2791-E from the Spanish Ministry of Science and Technology is acknowledged. TA would like to acknowledge the financial support from Secretaria de Estado de Educación y Universidades (SB2000-0166), the Spanish Ministry of Education, Culture and Sport.

\section{References}

[1] Namias V 1980 J. Inst. Math. Appl. 25241

[2] McBride A C and Kerr F H 1987 IMA J. Appl. Math. 39159

[3] Mendlovic D and Ozaktas H M 1993 J. Opt. Soc. Am. A 101875

[4] Almeida L B 1994 IEEE Trans. Signal Process. 423084 
[5] Ozaktas H M, Zalevsky Z and Kutay M A 2001 The Fractional Fourier Transform — with Applications in Optics and Signal Processing (Chichester: Wiley)

[6] Mendlovic D, Ozaktas H M and Lohmann A W 1995 Appl. Opt. 34303

[7] Mustard D 1998 J. Aust. Math. Soc. B 40257

[8] Akay O and Boudreaux-Bartels G F 2001 IEEE Trans. Signal Process. 49979

[9] Lohmann A W 1993 J. Opt. Soc. Am. A 102181

[10] Abramowitz M and Stegun I A 1965 Handbook of Mathematical Functions (New York: Dover) p 12

[11] Ozaktas H M, Arikan O, Kutay M A and Bozdagi G 1996 IEEE Trans. Signal Process. 442141 